\title{
Simultaneously measured signals in scanning probe microscopy with a needle sensor: Frequency shift and tunneling current
}

Ireneusz Morawski, and Bert Voigtländer

Citation: Review of Scientific Instruments 81, 033703 (2010);

View online: https://doi.org/10.1063/1.3321437

View Table of Contents: http://aip.scitation.org/toc/rsi/81/3

Published by the American Institute of Physics

\section{Articles you may be interested in}

Combined frequency modulated atomic force microscopy and scanning tunneling microscopy detection for multitip scanning probe microscopy applications

Review of Scientific Instruments 86, 123703 (2015); 10.1063/1.4936975

Ultra compact multitip scanning tunneling microscope with a diameter of $50 \mathrm{~mm}$

Review of Scientific Instruments 83, 033707 (2012); 10.1063/1.3694990

A nanopositioner for scanning probe microscopy: The KoalaDrive

Review of Scientific Instruments 83, 023703 (2012); 10.1063/1.3681444

Resistance and dopant profiling along freestanding GaAs nanowires

Applied Physics Letters 103, 143104 (2013); 10.1063/1.4823547

Low vibration laboratory with a single-stage vibration isolation for microscopy applications

Review of Scientific Instruments 88, 023703 (2017); 10.1063/1.4975832

Voltage preamplifier for extensional quartz sensors used in scanning force microscopy

Review of Scientific Instruments 82, 063701 (2011); 10.1063/1.3594103

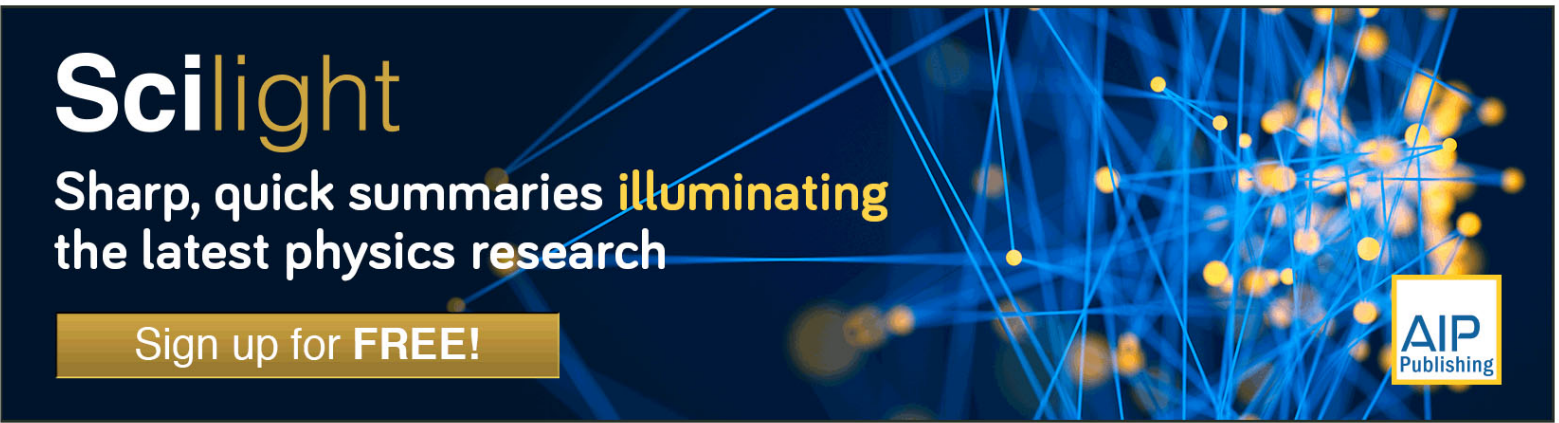




\title{
Simultaneously measured signals in scanning probe microscopy with a needle sensor: Frequency shift and tunneling current
}

\author{
Ireneusz Morawski ${ }^{1,2}$ and Bert Voigtländer ${ }^{1}$ \\ ${ }^{1}$ Institute of Bio- and Nanosystems, Forschungszentrum Jülich, 52425 Jülich, Germany \\ and JARA-Fundamentals of Future Information Technology, Forschungszentrum Jülich, \\ 52425 Jülich, Germany \\ ${ }^{2}$ Institute of Experimental Physics, University of Wroctaw, pl. M. Borna 9, 50-204 Wroctaw, Poland
}

(Received 23 November 2009; accepted 25 January 2010; published online 17 March 2010)

\begin{abstract}
We present combined noncontact scanning force microscopy and tunneling current images of a platinum(111) surface obtained by means of a $1 \mathrm{MHz}$ quartz needle sensor. The low-frequency circuit of the tunneling current was combined with a high-frequency signal of the quartz resonator enabling full electrical operation of the sensor. The frequency shift and the tunneling current were detected simultaneously, while the feedback control loop of the topography signal was fed using one of them. In both cases, the free signal that was not connected to the feedback loop reveals proportional-integral controller errorlike behavior, which is governed by the time derivative of the topography signal. A procedure is proposed for determining the mechanical oscillation amplitude by utilizing the tunneling current also including the average tip-sample work function. () 2010 American Institute of Physics. [doi:10.1063/1.3321437]
\end{abstract}

\section{INTRODUCTION}

A simultaneous measurement of atomic forces and tunneling current during imaging of surfaces is of great interest. $^{1,2}$ In order to simultaneously record both signals, a low oscillation amplitude of the quartz sensor is required, which should be close to the typical tip-sample interaction range that is in the order of a few angstroms or even lower. ${ }^{3,4}$ In order to fulfill this condition, it is advisable to use a quartz resonator with high stiffness and correspondingly high resonance frequency. Currently, the force sensor most frequently used to satisfy the abovementioned requirement is the tuning fork sensor. ${ }^{5}$ Here, we took a slightly different direction and decided to use the so-called needle sensor, ${ }^{6,7}$ which we believe has several advantages compared to the tuning fork sensor. Even smaller oscillation amplitudes should be accessible with the even higher stiffness of this sensor. Moreover, both the excitation and detection are fully integrated within the sensor itself, while for other sensors, usually, an external mechanical excitation of the oscillation is applied. In addition, as distinguished from the tuning fork, mechanical oscillations proceed along the needle-sensor axis, while its dimensions are comparable to the tuning fork quartz, or even smaller. These facts make the needle sensor also attractive for applications in which little space is available for the sensor, such as for multitip scanning probe microscopy. ${ }^{8-10}$ In this paper, we present scanning probe microscopy data obtained using a needle-sensor quartz resonator.

A quantitative analysis of the tip-sample interaction forces requires accurate knowledge of the oscillation amplitude. ${ }^{11-13}$ The amplitude can be determined using methods based on frequency shift dependency, ${ }^{14,15}$ charge, and thermal vibrations, ${ }^{15}$ as well as tunneling current dependency. ${ }^{16}$ Here, we concentrate on amplitude determination using the tunneling current, which we believe is the most reliable method. In an amplitude calibration method used in Ref. 16, the tip-sample work function is not taken into account. Here, we present an extension of this method including the work function determined either separately or in parallel with amplitude calibration.

\section{EXPERIMENTAL SETUP}

In the present work, the $1 \mathrm{MHz}$ quartz resonator with mechanical oscillation proceeding along the bar was applied as a scanning probe. A relatively high fundamental resonance frequency and a large value of the spring constant, in the order of $0.5 \mathrm{MN} / \mathrm{m},{ }^{17}$ enable an ultralow oscillation amplitude and a fast scan rate at reasonably low thermal frequency noise. ${ }^{18,19}$ The needle sensor was equipped with a platinumcoated silicon tip attached to the sensor by means of conductive glue, as well as electrically connected to one of the resonator electrodes. Tip-sample interaction force related to frequency shift $^{20}$ was detected in frequency modulation mode at constant amplitude by means of a phase-locked loop (PLL) controller. ${ }^{21}$ A $400 \mathrm{~Hz}$ bandwidth of the frequency shift demodulator was set for all the data presented here.

The circuit shown in Fig. 1 was used in order to simultaneously measure a frequency shift and a tunneling current. The tip-sample separation is controlled by a proportionalintegral controller (PI-c), ${ }^{22}$ which steers the Z-extension of the piezotube. ${ }^{23}$ Both tunneling current $\mathrm{I}_{\mathrm{T}}$ and frequency shift $\Delta \mathrm{f}$ are influenced by the tip-sample distance, providing a signal which can be used as a feedback signal for the PI-c. During scanning, the feedback was fed by one of these signals, making it possible to record the other one as a "free signal" (not used in the Z-loop). Rectifying the PI-c input signal ensures independence of the $\mathrm{I}_{\mathrm{T}}$ sign on the bias voltage as well as enabling measurements in the attractive force range. ${ }^{24}$ The measurement loop of the tunneling current was 


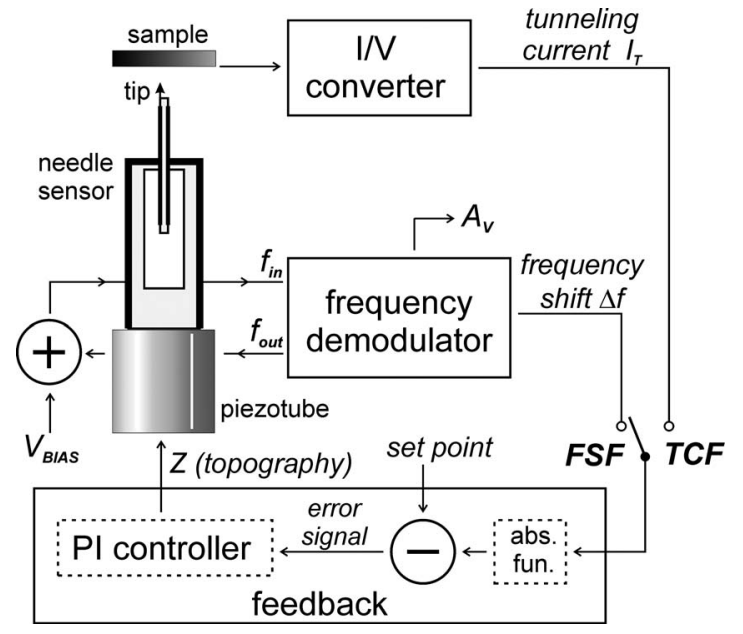

FIG. 1. Block diagram of simultaneous measurement of frequency shift and tunneling current in scanning probe microscopy. Signals: Z (topography), $\Delta \mathrm{f}$, and $\mathrm{I}_{\mathrm{T}}$ are recorded by PC software. The Z-feedback loop is fed by either frequency shift or tunneling current (position FSF or TCF, respectively), making it possible to log the latter as a free signal. The XY scan circuit is omitted in the diagram.

combined with a circuit feeding the quartz resonator by adding the bias voltage to the high-frequency signal driving the sensor. The $I / V$ converter bandwidth of about $1 \mathrm{kHz}$ sufficiently time averaged the $1 \mathrm{MHz}$ component. Moreover, electric separation is not needed due to the relatively low reactance (order of hundreds kilo-ohms) of a tip-sample capacity at resonance frequency, which is still a few orders of magnitude lower than the resistance usually present at the tunneling gap. This setup enables full electrical steering of the sensor as well as the electrical detection of measured signals.

The experiment was performed in an ultrahigh vacuum system equipped with a home-made scanning tunneling microscope (STM). ${ }^{25}$ We adapted a scanning beetle ${ }^{26}$ head to mount the needle sensor as a removable part. The $\mathrm{Pt}(111)$ bead monocrystal ${ }^{27,28}$ was prepared as usual by repeated cycles of long-term annealing at about $700{ }^{\circ} \mathrm{C}$, and then flashing at about $1100{ }^{\circ} \mathrm{C}$ directly before the experiment was performed. The easyPLL (from Nanosurf) system was used as the PLL controller in the frequency shift measurements circuit, while a Femto transimpedance amplifier was applied as the $I / V$ converter in the tunneling current measurement loop.

\section{AMPLITUDE CALIBRATION}

The tunneling-current-controlled mode was applied in order to determine the mechanical oscillation amplitude of the needle sensor. ${ }^{16}$ We extended this method in order to allow for a network function of the tip-surface system as well as to apply a relatively low oscillation amplitude of the probe. Following the procedure in Ref. 16, a relation between the mechanical oscillation $\mathrm{A}_{0}$ of the quartz sensor and the corresponding voltage amplitude $\mathrm{A}_{\mathrm{V}}$ detected in the frequency demodulator (Fig. 1) can be written as $\mathrm{A}_{0}=\alpha \mathrm{A}_{\mathrm{V}}$, where $\alpha$ is the amplitude calibration coefficient to be determined. The dependence of the tunneling current $\mathrm{I}_{\mathrm{T}}$ on the tip-sample displacement $\mathrm{Z}$ can be written as ${ }^{23}$

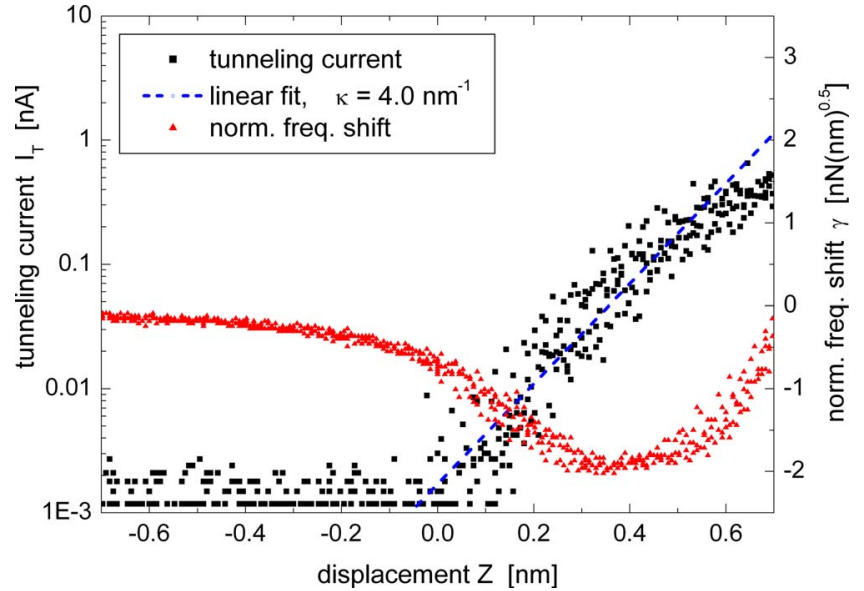

FIG. 2. (Color online) Normalized frequency shift $\gamma$ and tunneling current $\mathrm{I}_{\mathrm{T}}$ dependence on the tip-sample separation taken simultaneously for a $\mathrm{Pt}(111)$ sample at $\mathrm{V}_{\text {BIAS }}=0.5 \mathrm{~V}$ and constant amplitude mode with oscillation amplitude $\mathrm{A}_{0}=0.8 \mathrm{~nm}$. The exponential behavior of the $\mathrm{I}_{\mathrm{T}}(\mathrm{Z})$ function is marked by a dashed line with the slope coefficient $\kappa=4.0 \mathrm{~nm}^{-1}$.

$$
\mathrm{I}_{\mathrm{T}}(\mathrm{Z})=\mathrm{V}_{\mathrm{B}} \mathrm{Ke}^{-2 \kappa \mathrm{Z}}, \quad \kappa=\frac{\sqrt{2 \mathrm{~m}_{\mathrm{e}} \Phi}}{\mathrm{h}},
$$

where the coefficient $\kappa$ includes the average of the tip and sample work functions $\Phi,{ }^{29} \mathrm{~K}$ is a constant, and $\mathrm{V}_{\mathrm{B}}$ is a bias voltage. Further, the average tunneling current over one period of the sensor oscillation is calculated as follows:

$$
\left\langle\mathrm{I}_{\mathrm{T}}(\mathrm{Z})\right\rangle=\frac{1}{2 \pi} \int_{0}^{2 \pi} \mathrm{V}_{\mathrm{B}} \mathrm{Ke}^{-2 \kappa\left(\mathrm{Z}_{\mathrm{EQU}}+\mathrm{A}_{0} \sin \varphi\right)} \mathrm{d} \varphi
$$

Deriving the absolute equilibrium position $\mathrm{Z}_{\mathrm{EQU}}$ of the tip from above formula, one obtains

$$
\begin{aligned}
\mathrm{Z}_{\mathrm{EQU}} & =\frac{1}{2 \kappa} \ln \left(\frac{\mathrm{V}_{\mathrm{B}} \mathrm{K}}{\left\langle\mathrm{I}_{\mathrm{T}}\right\rangle} \frac{1}{2 \pi} \int_{0}^{2 \pi} \mathrm{e}^{-2 \kappa \mathrm{A}_{0} \sin \varphi} \mathrm{d} \varphi\right) \\
& =\frac{1}{2 \kappa} \ln \left(\frac{\mathrm{V}_{\mathrm{B}} \mathrm{K}}{\left\langle\mathrm{I}_{\mathrm{T}}\right\rangle}\right)+\Delta \mathrm{Z}_{\mathrm{EQU}}\left(\mathrm{A}_{0}, \kappa\right) .
\end{aligned}
$$

The $Z_{\mathrm{EQU}}$ consists of two terms, where only one contains the oscillation amplitude $\mathrm{A}_{0}$, namely, the displacement $\Delta \mathrm{Z}_{\mathrm{EQU}}$ from the equilibrium position

$$
\Delta \mathrm{Z}_{\mathrm{EQU}}\left(\mathrm{A}_{0}, \kappa\right)=\frac{1}{2 \kappa} \ln \left(\frac{1}{2 \pi} \int_{0}^{2 \pi} \mathrm{e}^{-2 \kappa \mathrm{A}_{0} \sin \varphi} \mathrm{d} \varphi\right) .
$$

$\Delta \mathrm{Z}_{\mathrm{EQU}}$ is independent of $\left\langle\mathrm{I}_{\mathrm{T}}\right\rangle$ and equals 0 for $\mathrm{A}_{0}=0$.

In the following, we describe two ways of extending the method presented in Ref. 16. First, the value of $\alpha$ can be determined with previous knowledge of $\kappa$ that is related to the work function. Second, $\alpha$ and also $\kappa$ are evaluated simultaneously using a matching of the theoretical relation (4) to the experimental data.

The factor $\kappa$ can be determined from a direct measurement of $\mathrm{I}_{\mathrm{T}}(\mathrm{Z})$, as presented in Fig. 2. Introducing a dimensionless quantity $\xi=2 \kappa \mathrm{A}_{0}$, Eq. (4) can be written as follows: 


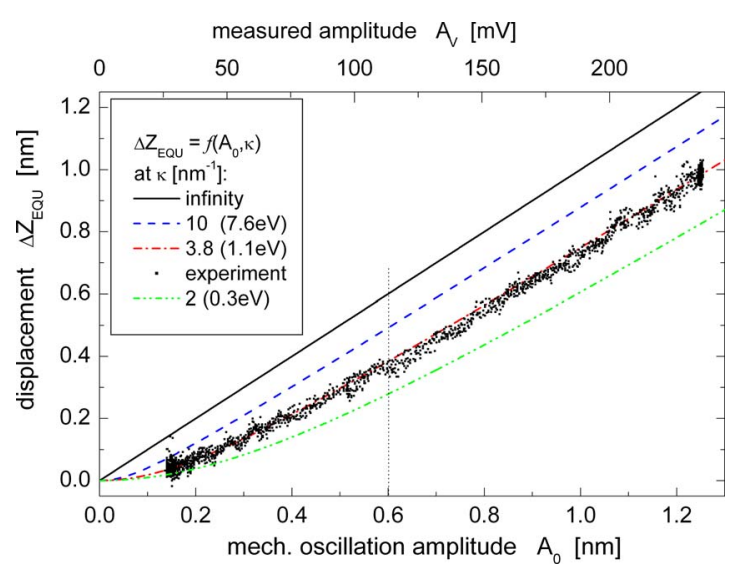

FIG. 3. (Color online) Dependence of the displacement $\Delta \mathrm{Z}_{\mathrm{EQU}}$ from the equilibrium tip-sample position [Eq. (4)] vs the oscillation amplitude $A_{0}$ as well as the readout voltage amplitude $A_{V}$ for different values of the parameter $\kappa$. At low values of $\mathrm{A}_{0}$ or $\kappa$, i.e., $2 \kappa \mathrm{A}_{0} \sim 1$, the slope deviates substantially from unity. For instance, at $\mathrm{A}_{0}=0.6 \mathrm{~nm}$ the slope deviates by $25 \%$, $11 \%$, and $5 \%$ for $\kappa=2,4$, and $10 \mathrm{~nm}^{-1}$, respectively. The experimental data (black dots) were used to fit the $\Delta \mathrm{Z}_{\mathrm{EQU}}\left(\mathrm{A}_{0}, \kappa\right)$ curve (see dashed-dotted line).

$$
2 \kappa \Delta \mathrm{Z}_{\mathrm{EQU}}=\ln \left[\frac{1}{2 \pi} \int_{0}^{2 \pi} \mathrm{e}^{-\xi \sin \varphi} \mathrm{d} \varphi\right] \equiv f(\xi) .
$$

The value of the quantity $\xi$ is determined from the inverse function to $f$

$$
\xi=f^{-1}\left(2 \kappa \Delta \mathrm{Z}_{\mathrm{EQU}}\right) \text {. }
$$

The previous equation can be solved numerically using, for instance, a chart or a table. In this work, a simple "zerofinding" MATLAB ${ }^{\mathrm{TM}}$ script was used to evaluate $\xi$. Note that the dimensionless quantity $2 \kappa \Delta \mathrm{Z}_{\mathrm{EQU}}$ is the argument of the above function, not the directly measured value of $\Delta \mathrm{Z}_{\mathrm{EQU}}$. Returning to a dimensional quantity, $\mathrm{A}_{0}$ can be written as

$$
\mathrm{A}_{0}=\alpha \mathrm{A}_{\mathrm{V}}=\frac{f^{-1}\left(2 \kappa \Delta \mathrm{Z}_{\mathrm{EQU}}\right)}{2 \kappa} .
$$

Hence, $\alpha$ can be written as

$$
\alpha=\frac{f^{-1}\left(2 \kappa \Delta \mathrm{Z}_{\mathrm{EQU}}\right)}{2 \kappa \mathrm{A}_{\mathrm{V}}} .
$$

The value of the $\mathrm{Z}$-displacement $\Delta \mathrm{Z}_{\mathrm{EQU}}$ from the equilibrium tip position (for $A_{0}=0$ ) was recorded for a given voltage amplitude $A_{V}$ by reading it out from the scanning force microscope (SFM) control electronics. Using a set of measured values, $\Delta \mathrm{Z}_{\mathrm{EQU}}=0.32 \mathrm{~nm}$ at $\mathrm{A}_{\mathrm{V}}=100 \mathrm{mV}$ as well as $\kappa$ $=4.0 \mathrm{~nm}^{-1}$, determined independently, the voltagemechanical coefficient $\alpha=5.8 \mathrm{~nm} / \mathrm{V}$ was calculated.

Here, we treat $\kappa$ as an unknown parameter and determine it from a fitting to experimental data in the course of the amplitude determination. The displacement $\Delta \mathrm{Z}_{\mathrm{EQU}}$ calculated from Eq. (4) is plotted in Fig. 3 as a function of the oscillation amplitude $\mathrm{A}_{0}$ for different values of $\kappa$. For a sufficiently large amplitude, i.e., $2 \kappa \mathrm{A}_{0} \gg 1$, the slope of $\Delta \mathrm{Z}_{\mathrm{EQU}}\left(\mathrm{A}_{0}, \kappa\right)$ approaches unity for all $\kappa$, allowing us to obtain the value of the coefficient $\alpha$ irrespective of $\kappa \cdot{ }^{16}$ However, for low values of $\mathrm{A}_{0}$ or $\kappa$, i.e., $2 \kappa \mathrm{A}_{0} \sim 1$, the slope deviates substantially from unity. For instance, at $\mathrm{A}_{0}$
$=0.6 \mathrm{~nm}$, the slope deviates from unity by $25 \%, 11 \%$, and $5 \%$ for $\kappa=2,4$, and $10 \mathrm{~nm}^{-1}$, respectively. A procedure avoiding the abovementioned inaccuracy in the determination of $\alpha$ is proposed in the following. Experimental data taken in a range of the readout voltage amplitude $A_{V}$ from 25 to $210 \mathrm{mV}$ at $\left\langle\mathrm{I}_{\mathrm{T}}\right\rangle=20 \mathrm{pA}$ and $\mathrm{V}_{\text {bias }}=0.8 \mathrm{~V}$ are shown in Fig. 3 as black dots. The theoretical dependence $\Delta \mathrm{Z}_{\mathrm{EQU}}$ $=\mathrm{f}\left(\alpha \mathrm{A}_{\mathrm{V}}, \kappa\right)$ is fitted to the experimental data for the two sought parameters $\alpha$ and $\kappa$. The function $\Delta \mathrm{Z}_{\mathrm{EQU}}$ $=\mathrm{f}\left(\alpha \mathrm{A}_{\mathrm{V}}, \kappa\right)$ is monotonic both for $\alpha$ and $\kappa$. It ensures that for the increment in $\Delta \mathrm{Z}_{\mathrm{EQU}}$ obtained in the given range of readout voltage amplitude $\mathrm{A}_{\mathrm{V}}$, there is only one pair $(\alpha, \kappa)$ giving the best theoretical values fitting the experiment. The fitting results in $\kappa=(3.83 \pm 0.05) \mathrm{nm}^{-1}$ and $\alpha=(5.9 \pm 0.05) \mathrm{nm} / \mathrm{V}$ for the data showed in Fig. 3, which is in good agreement with the directly evaluated value of $\kappa=0.4 \mathrm{~nm}^{-1}$ (Fig. 2) as well as with $\alpha=5.8 \mathrm{~nm} / \mathrm{V}$ previously obtained. The upper abscissa axis in Fig. 3 shows the readout voltage amplitude $\mathrm{A}_{\mathrm{V}}$ of the probe oscillation using the coefficient $\alpha$ estimated above. Note that the quantity $A_{V}$ is strictly dependent on the given electronic circuit as distinct from $\mathrm{A}_{0}$. The procedures presented above avoid the necessity of using large oscillation amplitudes and, in the case of the fitting procedure, an independent experimental determination of the tip-sample work function.

\section{RESULTS}

Figure 2 shows the normalized frequency shift $\gamma$ $=\left(\Delta \mathrm{f} / \mathrm{f}_{0}\right) \mathrm{kA}_{0}^{3 / 2}$ (Ref. 30) and tunneling current dependences on the tip-sample distance taken simultaneously for the platinum sample at $\mathrm{V}_{\text {BIAS }}=0.5 \mathrm{~V}$ with the feedback loop switched off. The high stiffness $\mathrm{k}$ of quartz of about 0.5 $\mathrm{MN} / \mathrm{m}$ (Ref. 17) enables a measurement with relatively low oscillation amplitude $\mathrm{A}_{0}$, here $0.8 \mathrm{~nm}$, while maintaining a low intrinsic frequency noise. ${ }^{18,19}$ Although the needlesensor stiffness is a few orders of magnitude larger than the typical interatomic force gradient, the ratio of the resonance frequency $\mathrm{k}$ and $\mathrm{f}_{0}$ (Ref. 30) is in the order of unity, here equal to $0.54 \mathrm{nNHz} / \mathrm{nm}$, thus providing values of the frequency shift comparable to other SFM probes with much lower stiffness, such as cantilevers or tuning forks. ${ }^{31}$ The typical minimum of the $\gamma(\mathrm{Z})$ dependence is well recognized with the value of about $-2 \mathrm{nN}(\mathrm{nm})^{0.5}$, providing a stable scanning process also in the attractive tip-sample force range. The exponential part of the tunneling current relation (with currents above the inner noise level of the $I / V$ converter) was fitted by the exponential function with the coefficient $\kappa=(4.0 \pm 0.15) \mathrm{nm}^{-1}$. We chose the point at which the tunneling current rises above the noise level as the zero point for the Z-displacement.

Images of the platinum(111) surface investigated using a quartz needle sensor are shown in Fig. 4. The multilayer atomic steps can be easily recognized in the topography image. The $\mathrm{Z}$ noise was about $0.2 \AA$ with the related lateral resolution of about $10 \mathrm{~nm} .^{32}$ The feedback loop was closed in the frequency shift feeds (FSF) position, in which the frequency shift output of demodulator electronics is fed as input into the feedback circuit, delivering an error signal for the 


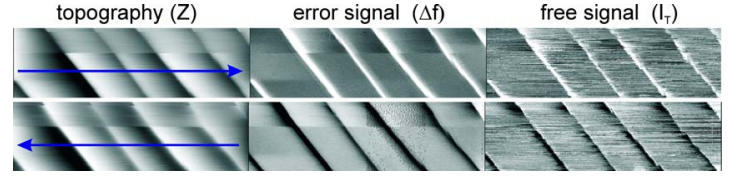

FIG. 4. (Color online) A scan of a platinum(111) surface taken in the FSF mode (Z-feedback loop). Upper and lower panel show forward and backward scans of the same area, respectively. The arrows indicate the direction of tip motion during scanning. The corresponding line profiles are shown in Fig. 5. The width of the scan area is $400 \mathrm{~nm}$, set point: $-1.6 \mathrm{nN}(\mathrm{nm})^{-0.5},(3$ $\mathrm{Hz}$ ); oscillation amplitude: $1.0 \mathrm{~nm}$; and acquisition speed: $1.5 \mathrm{lps}$.

PI-c. The simultaneously recorded tunneling current was not used for the feedback. Therefore, this signal is termed the free signal. Both signals are shown in the middle and righthand images in Fig. 4. Here, we intentionally set the proportional parameter of the PI-c to a low value. ${ }^{33}$ This leads to an overshoot in the error signal when scanning in the step-up direction, as also seen in Fig. 5. The free signal follows this error signal because both are related to the distance between the tip apex and the surface. On the other hand, this distance is controlled by the PI-c and differs greatly from the set point value near steps due to the slow PI-c response during scanning. Applying a simple model for the PI-c and assuming a proportional transfer function of the other parts of the loop, i.e., the high-voltage converter, the Z-piezotube, and the frequency demodulator ( $I / V$ converter), the Z-topography signal can be written as

$$
\mathrm{z}(\mathrm{t})=\operatorname{Pe}(\mathrm{t})+\frac{1}{\mathrm{~T}} \int_{0}^{\mathrm{t}} \mathrm{e}(\tau) d \tau
$$

where $\mathrm{e}(\mathrm{t})$ is the error signal, and $\mathrm{P}$ and $\mathrm{T}$ are proportional and integral parameters, respectively. Hence, for sufficiently low values of $\mathrm{P}$, the following error signal results:

$$
\mathrm{e}(\mathrm{t}) \propto \mathrm{T} \frac{\mathrm{dz}(\mathrm{t})}{\mathrm{dt}} .
$$

The error signal is proportional to the time derivative of the topography signal. This is in good agreement with the experimental profiles shown in Fig. 5, where the correlation of

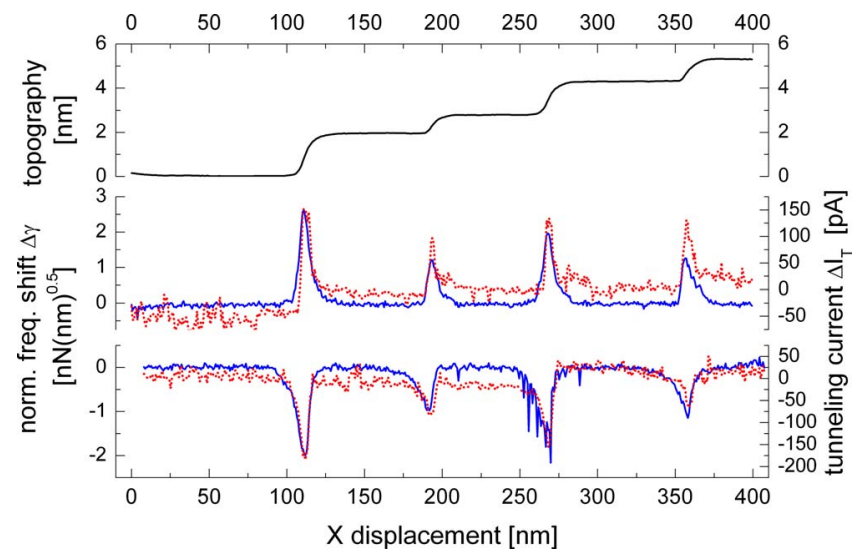

FIG. 5. (Color online) Line profile of the topography (upper panel). The middle and the lower panels show the variation around the working point of the error signal (normalized $\Delta \mathrm{f}$ ) as solid lines, and the tunneling current $I_{T}$ recorded as the free signal as dotted lines for data recorded in the forward and backward directions, respectively. The profiles were taken along the arrows marked in Fig. 4.

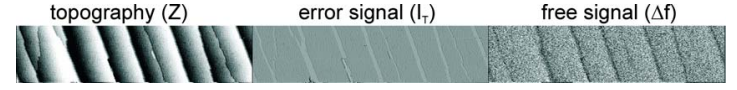

FIG. 6. (Color online) A scan of the platinum surface taken at the TCF position. The PI-c was fed by tunneling current $\mathrm{I}_{\mathrm{T}}$, the free signal (here, frequency shift $\Delta \mathrm{f}$ ) follows the error signal with a minus sign. A scan area width is $250 \mathrm{~nm}$; set point: $10 \mathrm{pA}$; the oscillation amplitude: $2.3 \mathrm{~nm}$; and the acquisition speed: $3.0 \mathrm{lps}$.

the error peak height with the height of particular atomic steps is readily visible.

Although the scans presented here were taken in the attractive force regime, the streaks observed in the tunneling current images (right-hand column in Fig. 4) probably result from a momentary contact between tip and sample due to the slow PI-c response.

Finally, measurements in the tunneling current feedback (TCF) mode were performed as shown in Fig. 6. In this case, the tunneling current fed the feedback loop and the frequency shift was recorded as a free signal. Strips related to atomic steps are distinguished in both the error signal and the free signal pattern. However, in contrast to the case discussed above, the latter has the opposite sign to the former. The frequency shift dependence is not a monotone function of the tip-sample distance, and when acquired as a free signal in the attractive force range, its derivative has the opposite sign to the tunneling current derivative (see Fig. 2). Therefore, positive deviations in the error signal (tunneling current) at step edges correspond to negative ones in the frequency shift signal.

\section{CONCLUSIONS}

We performed scanning probe microscopy investigations on a platinum surface by means of a $1 \mathrm{MHz}$ extensional quartz sensor, as yet rarely used as an SFM sensor. An electronic circuit for simultaneous measurement of frequency shift and tunneling current was proposed in this paper. Using a current/voltage converter with a relatively narrow bandwidth in comparison to the driving frequency of the quartz, a common electric circuit for simultaneous operation in both STM and SFM mode is possible. The determination of mechanical oscillation amplitude utilizing the tunneling-currentcontrolled procedure was extended to include the determination of the average tip-sample work function. In addition, we showed that the probe signal, which is not used for the Z-feedback, follows the error signal of the PI-c.

\section{ACKNOWLEDGMENTS}

We would like to thank Vasily Cherepanov for many helpful discussions, and Helmut Stollwerk and Peter Coenen for their expert technical assistance.

${ }^{1}$ T. Eguchi, Y. Fujikawa, K. Akiyama, T. An, M. Ono, T. Hashimoto, Y. Morikawa, K. Terakura, T. Sakurai, M. G. Lagally, and Y. Hasegawa, Phys. Rev. Lett. 93, 266102 (2004).

${ }^{2}$ H. Ö. Özer, S. J. O’Brien, and J. B. Pethica, Appl. Phys. Lett. 90, 133110 (2007)

${ }^{3}$ A. Oral, R. A. Grimble, H. Ö. Özer, P. M. Hoffmann, and J. B. Pethica, Appl. Phys. Lett. 79, 1915 (2001).

${ }^{4}$ M. Herz, Ch. Schiller, F. J. Giessibl, and J. Mannhart, Appl. Phys. Lett. 86, 153101 (2005). 
${ }^{5}$ F. J. Giessibl, Appl. Phys. Lett. 73, 3956 (1998).

${ }^{6}$ U. Grunewald, K. Bartzke, and T. Antrack, Thin Solid Films 264, 169 (1995).

${ }^{7}$ S. Heike and T. Hashizume, Appl. Phys. Lett. 83, 3620 (2003).

${ }^{8}$ R. Hobara, N. Nagamura, S. Hasegawa, I. Matsuda, Y. Yamamoto, Y. Miyatake, and T. Nagamura, Rev. Sci. Instrum. 78, 053705 (2007).

${ }^{9}$ P. Jaschinsky, P. Coenen, G. Pirug, and B. Voigtländer, Rev. Sci. Instrum. 77, 093701 (2006).

${ }^{10}$ P. Jaschinsky, J. Wensorra, M. I. Lepsa, J. Myslivecek, and B. Voigtländer, J. Appl. Phys. 104, 094307 (2008).

${ }^{11}$ J. E. Sader and S. P. Jarvis, Appl. Phys. Lett. 84, 1801 (2004).

${ }^{12}$ M. A. Lantz, H. J. Hug, R. Hoffmann, P. J. A. van Schendel, P. Kappenberger, S. Martin, A. Baratoff, and H.-J. Güntherodt, Science 291, 2580 (2001).

${ }^{13}$ F. J. Giessibl, Appl. Phys. Lett. 78, 123 (2001)

${ }^{14}$ W. Clauss, J. Zhang, D. J. Bergeron, and A. T. Johnson, J. Vac. Sci. Technol. B 17, 1309 (1999).

${ }^{15}$ F. J. Giessibl, Appl. Phys. Lett. 76, 1470 (2000).

${ }^{16}$ G. H. Simon, M. Heyde, and H.-P. Rust, Nanotechnol. 18, 255503 (2007).

${ }^{17}$ Z. Peng and P. West, Appl. Phys. Lett. 86, 014107 (2005).

${ }^{18}$ T. R. Albrecht, P. Grütter, D. Horne, and D. Rugar, J. Appl. Phys. 69, 668 (1991).

${ }^{19}$ U. Dürig, O. Züger, and A. Stalder, J. Appl. Phys. 72, 1778 (1992).
${ }^{20}$ R. García and R. Perez, Surf. Sci. Rep. 47, 197 (2002).

${ }^{21}$ U. Dürig, H. R. Steinauer, and N. Blanc, J. Appl. Phys. 82, 3641 (1997).

${ }^{22}$ H. L. Wade, Basic and Advanced Regulatory Control: System Design and Application, 2nd ed. (ISA, Research Triangle Park, 2004).

${ }^{23}$ C. J. Chen, Introduction to Scanning Tunneling Microscopy (Oxford University Press, New York, 2008)

${ }^{24}$ M. Heyde, M. Sterrer, H.-P. Rust, and H.-J. Freund, Appl. Phys. Lett. 87, 083104 (2005).

${ }^{25}$ B. Voigtländer, Surf. Sci. Rep. 43, 127 (2001).

${ }^{26}$ K. Besocke, Surf. Sci. 181, 145 (1987).

${ }^{27}$ B. Voigtländer, U. Linke, H. Stollwerk, and J. Brona, J. Vac. Sci. Technol. A 23, 1535 (2005).

${ }^{28}$ B. Voigtländer, V. Cherepanov, Ch. Elsaesser, and U. Linke, Rev. Sci. Instrum. 79, 033911 (2008).

${ }^{29}$ N. W. Ashcroft and N. D. Mermin, Solid State Physics (Saunders College, Philadelphia, 1976).

${ }^{30}$ F. J. Giessibl, Phys. Rev. B 56, 16010 (1997).

${ }^{31}$ F. J. Giessibl, Rev. Mod. Phys. 75, 949 (2003).

${ }^{32}$ R. Nishi, I. Houda, T. Aramata, Y. Sugawara, and S. Morita, Appl. Surf. Sci. 157, 332 (2000).

${ }^{33}$ G. Ellis, Control System Design Guide, 3rd ed. (Elsevier Academic, London, 2004. 